\title{
External calibration with Drosophila whole-cell spike-ins delivers absolute mRNA fold changes from human RNA-Seq and qPCR data
}

\author{
Franziska Taruttis ${ }^{1 \star}$, Maren Feist² ${ }^{2 \star}$ Phillip Schwarzfischer ${ }^{3}$, Wolfram Gronwald ${ }^{3}$, Dieter Kube², \\ Rainer Spang ${ }^{1}$, and Julia C. Engelmann ${ }^{1}$ \\ 'Statistical Bioinformatics, University of Regensburg, Regensburg, Germany, ${ }^{2}$ Haematology and Oncology, \\ University Medical Center Göttingen, Göttingen, Germany, and ${ }^{3}$ Institute of Functional Genomics, University \\ of Regensburg, Regensburg, Germany
}

${ }^{*}$ F.T. and M.F. contributed equally to this work.

BioTechniques 62:53-61 (February 2017) doi 10.2144/000114514

Keywords: RNA sequencing (RNA-Seq); qPCR, gene expression; spike-in; external reference; calibration

Supplementary material for this article is available at www.BioTechniques.com/article/114514.

Gene expression measurements are typically performed on a fixed-weight aliquot of RNA, which assumes that the total number of transcripts per cell stays nearly constant across all conditions. In cases where this assumption does not hold (e.g., when comparing cell types with different cell sizes) the expression data provide a distorted view of cellular events. Assuming constant numbers of total transcripts, increases in expression of some RNAs must be compensated for by decreases in expression of others. Therefore, we propose calibrating gene expression data to an external reference point, the number of cells in the sample, using whole-cell spike-ins. In a systematic dilution experiment, we mixed varying numbers of human cells with fixed numbers of Drosophila melanogaster cells and scaled the expression levels of the human genes relative to those of the Drosophila genes. This approach restored the original gene expression ratios generated by the dilutions. We then used Drosophila whole-cell spike-ins to uncover non-symmetric gene expression changes, in this case much larger numbers of induced than repressed genes, under perturbations of the human cell line P493-6. Drosophila whole-cell spike-ins are an experimentally and computationally easy and low-priced method to derive mRNA fold changes of absolute abundances from RNA sequencing (RNA-Seq) and quantitative real-time PCR (qPCR) data.

For decades, total RNA amounts per cell were assumed to be nearly constant between different physiological conditions. This assumption directly influenced how gene expression analysis was performed using methods such as quantitative real-time PCR (qPCR), microarrays, and RNA sequencing (RNA-Seq), as all protocols start with a fixed-weight aliquot of RNA. In spite of the constant amount of input material, technical variation in cell lysis, RNA extraction, and data generation results in variable library sizes or total array intensities. While qPCR data are typically normalized to an internal standard (a housekeeping gene), technical variations in high-throughput data are corrected by computational normalization methods $(1,2)$. In practice, a constant amount of starting material is needed for these methods to justify the normalization that brings all samples to a common scale. However, there is increasing evidence that the amount of RNA per cell can change drastically between experimental conditions. Buettner et al. (3) reported differences in the RNA content of cells in different stages of the cell cycle and pointed out that these differences obscure studies of cellular perturbations. In some B-cell lines, induction of the transcription factor MYC leads to the induction of virtually all transcribed genes by a factor of two to three, accompanied with an increase in cell size $(4,5)$. Similar transcriptional up-regulation has been described for lipopolysaccharide (LPS) stimulation of $B$ cells by a process independent of MYC activation (6). Moreover, heat

\section{METHOD SUMMARY}

We introduce whole cells of Drosophila melanogaster as suitable spike-ins to calibrate human gene expression data to the number of cells in the sample, resulting in absolute fold-change estimates. 
shock and serum starvation can trigger global changes in the RNA content of mammalian cells (7). Global up-regulation of gene expression most likely also affects housekeeping gene expression in qPCR measurements, explaining the difficulty in finding genes with constant expression levels in different cell types and under different experimental conditions.

Whenever global gene expression changes occur upon treatment (or, for example, gene knockout), relative expression values introduce artificial dependencies between the genes. If total RNA content is assumed to be constant, every increase in the expression of one gene must be compensated for by a decrease in the expression of other genes. Although not reported on a 0-100 scale, relative expression values reflect percentages of the total transcriptome. Imagine that the transcripts of a gene constitute $0.1 \%$ of the transcriptome for cells of type $A$ and $0.2 \%$ for cells of type $\mathrm{B}$, corresponding to a 2-fold difference in relative abundance. If the amount of total RNA in $A$ is 3 times that of $B, 0.2 \%$ of the transcripts in cell type $B$ is actually less in terms of absolute numbers than $0.1 \%$ of the transcripts in cell type $A$. Expression values calibrated to an external reference point, such as the number of cells in the sample, do not reflect percentages and do not add up to a constant number. Instead, they can be used to calculate fold changes of absolute mRNA abundances.

The importance of quantifying global gene expression changes has been recognized in recent years $(8,9)$. Protocols for calibrating gene expression values to the number of sample cells are based on adding synthetic RNA to the total RNA of a quantified number of sample cells (9), or adding RNA from a different organism with high sequence divergence as a spike-in control (10). However, variations in cell lysis and RNA extraction efficiency between samples will affect the accuracy of the sample scaling factors derived from the RNA spike-ins.

Here, we introduce a whole-cell spike-in assay that allows calibration of gene expression values to the total number of cells. It is based on mixing a fixed number of Drosophila melanogaster reference cells with a fixed number of human sample cells prior to RNA extraction and calibrating the sample gene expression data with the Drosophila data. Our approach was inspired by Sun et al. (11), who introduced
A

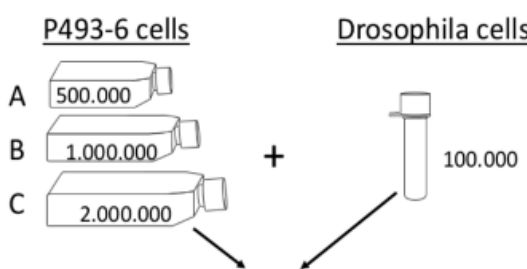

Mix at

harvest $\downarrow$

Lysis t

RNA isolation

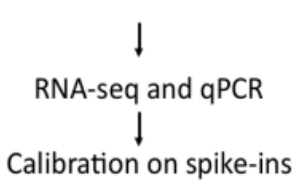

D

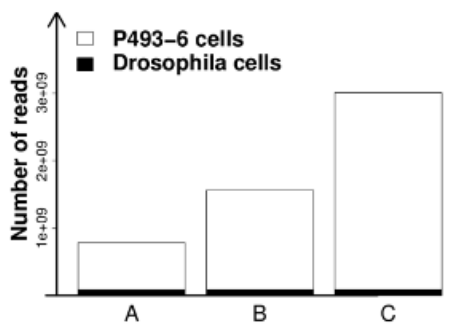

Figure 1. Isolation of RNA from different numbers of human cells and constant numbers of Drosophila melanogaster spike-in cells simulates changes in total RNA amounts per cell. (A) Experimental setup. Condition A: 500,000 cells from human B-cell line P493-6; Condition B: 1,000,000 P493-6 cells; and Condition C: 2,000,000 P493-6 cells. Samples were spiked with 100,000 S2 D. melanogaster cells, mixed, lysed, and total RNA was extracted. RNA was subjected to RNA sequencing (RNA-Seq) and quantitative real-time PCR (qPCR). Each condition (A, B, and C) consisted of three replicate samples. (B) Different cell numbers spiked with constant numbers of $D$. melanogaster cells simulate non-symmetric gene expression changes. The global ratios were 2:1 between Conditions $B$ and $A$ as well as between Conditions $C$ and $B$, and $4: 1$ between Conditions $C$ and $A$. (C) After taking a fixed-weight aliquot of the total RNA consisting of human and D. melanogaster transcripts for sequencing, the fraction of reads originating from $D$. melanogaster transcripts inversely correlated with the amount of human total RNA. (D) After calibration of human gene expression levels to D. melanogaster gene expression levels, the original ratios between Conditions $\mathrm{A}, \mathrm{B}$, and $\mathrm{C}$ were restored.

labeled whole-cell spike-ins in the context of dynamic expression changes in yeast using custom microarrays. We transferred the basic idea to RNA-Seq and qPCR technology, showing in a systematic dilution experiment that $D$. melanogaster is a suitable spike-in organism for human samples, and that whole cell-calibrated data produced more accurate fold-change estimates than data calibrated using synthetic RNA spike-ins.

\section{Materials and methods}

Cell culture and cell spike-in

Schneider S2 cells derived from $D$. melanogaster were cultured in Gibco Schneider's Drosophila medium (ThermoFisher Scientific, Waltham, MA) supplemented with 10\% FCS (ThermoFisher
Scientific) and penicillin/streptomycin. Cells were grown at room temperature and split once to twice a week. For spike-in preparation, S2 cells were counted with a hemocytometer, and aliquots of cells were frozen in $1 \mathrm{~mL}$ freezing media [45\% conditioned S2-media, 45\% FCS, 10\% DMSO (all percentages are volume/ volume)]. Spike-in stocks were stored at $-150^{\circ} \mathrm{C}$. All samples belonging to one experiment were spiked with cells from the same stock. The human B-cell line P493-6, carrying a conditional tetracycline/doxycycline-regulated MYC gene, a kind gift from Georg Bornkamm (Munich, Germany), was cultured in RPMI medium (Lonza, Basel, Switzerland) supplemented with $10 \%$ tetracycline-free FCS (Lonza) and penicillin/streptomycin at $37^{\circ} \mathrm{C}$ and $5 \% \mathrm{CO}_{2}$. For suppression of $\mathrm{MYC}$ 
expression, cells were treated with $1 \mathrm{ng} /$ $\mathrm{mL}$ doxycycline for $16 \mathrm{~h}$ (Myc ${ }^{\text {low }}$ condition). For the Mychigh condition, cells were kept in RPMI medium without doxycycline. The comparison between $\mathrm{Myc}^{\text {high }}$ and $\mathrm{Myc}^{\text {low }}$ consisted of 10 biological replicates each; the dilution experiment had 3 biological replicates per group. For the stimulation experiments, Myc ${ }^{\text {low }}$ cells were treated with $130 \mathrm{ng} / \mathrm{mL} \alpha$-IgM F(ab)2 fragments (Jackson ImmunoResearch, Suffolk, UK), $100 \mathrm{ng} / \mathrm{mL}$ soluble CD40 ligand (sCD40L; Autogen Bioclear, Nottingham, UK), or 0.5 $\mu \mathrm{M}$ CpG oligodeoxynucleotide (ODN2006; Invivogen, San Diego, CA) for $24 \mathrm{~h}$ in 2 biological replicates. Cells were seeded in fresh media at a density of $10^{6}$ cells/mL $24 \mathrm{~h}$ before harvest. For RNA isolation, P493-6 cells were counted with a hemocytometer, and a sample with the indicated number of cells (see Figure 1) was transferred into a centrifugation tube. For each experiment, a fresh aliquot of $\mathrm{S} 2$ cells was thawed for 2 min in a $37^{\circ} \mathrm{C}$ water bath. Keeping the spike-in cells in suspension, $20 \mu \mathrm{l}(=100,000$ cells) S2 cells was added directly to each transferred P493-6 cell suspension. Together, cells were centrifuged for 5 min at $900 \times g$ and washed once with cold PBS. Dry pellets were stored at $-80^{\circ} \mathrm{C}$. A detailed protocol for preparation and use of Drosophila whole-cell spike-ins is included in the Supplementary Material.

RNA isolation and ERCC RNA spike-in Cell pellets were lysed, and total RNA was extracted using the NucleoSpin RNA Isolation Kit (Machery-Nagel, Düren, Germany) according to the manufacturer's protocol. Total RNA was extracted from columns using $50 \mu \mathrm{l}$ RNase-free water and spiked with $2 \mu$ of a 1:100 dilution of External RNA Control Consortium (ERCC) RNA Spike-In Mix 1 (Life Technologies, now ThermoFisher Scientific) (12). Quantity and quality of the RNA were assessed using a Nanodrop 1000 (ThermoFisher Scientific) and Bioanalyzer 2100 (Agilent, Santa Clara, CA), respectively.

Quantitative real-time PCR (qPCR) For qPCR, RNA was transcribed to cDNA using Invitrogen SuperScript II Reverse Transcriptase (ThermoFisher Scientific) and random hexamer primers (IBA, Göttingen, Germany). cDNA samples were analyzed by SYBR Green-based qPCR using the Applied Biosystems $7900 H T$ Fast Real-Time PCR System (ThermoFisher Scientific). $\Delta \mathrm{C}_{\mathrm{q}}$ values were normalized to either Drosophila Act42A expression (spike-in cells) or expression of the human internal housekeeper gene GAPDH (P493-6 cells). Primer sequences are listed in Supplementary Table S1.

\section{RNA sequencing}

RNA-Seq libraries were prepared from $1 \mu \mathrm{g}$ total RNA containing RNA from the human cells, the Drosophila spike-in cells, and the ERCC Spike-In Mix 1. Library preparation was done using the TruSeq RNA Sample Preparation Kit v2 (Illumina, San Diego, CA). Libraries were sequenced single-end for 100 cycles on an Illumina HiSeq 2000. Raw sequence data are available through the NCBI BioProject database (www. ncbi.nlm.nih.gov/bioproject/): accession numbers PRJNA311567 (dilution experiment) and PRJNA312050 (MYC experiments).

\section{Preparation of a custom genome}

We concatenated the genomes of Homo sapiens (GRCh38, Ensembl release 77) (13), D. melanogaster (Ensembl BDGP5, release 77 ), and the ERCC sequences (https://tools.lifetechnologies.com/ content/sfs/manuals/cms_095047. txt). Sequence libraries were mapped against this concatenated genome using TopHat version 2.0.13 (14), indicating an unstranded sequencing protocol (-library-

Table 1: Number of differentially expressed genes based on endogenously or externally calibrated gene expression data.

\begin{tabular}{|l|l|l|l|l|} 
Stimulus & \multicolumn{2}{l}{ Calibration } & \multicolumn{1}{l}{$\begin{array}{l}\text { Number of differentially } \\
\text { expressed genes }\end{array}$} & $\begin{array}{l}\text { Number of } \\
\text { induced genes }\end{array}$ \\
\hline BCR & Endogenous & 2508 & 1273 & 1235 \\
\hline & External & 1694 & 1688 & 6 \\
\hline CD4OL & Endogenous & 1128 & 576 & 552 \\
\hline & External & 1 & 1 & 0 \\
\hline CpG & Endogenous & 4602 & 2342 & 2260 \\
\hline & External & 5570 & 5457 & 113 \\
\hline
\end{tabular}

Genes with adjusted $P$-values $<0.05$ were considered significantly differentially expressed between the stimulated cells and unstimulated controls (see "Materials and methods" section). $n=10$ for unstimulated controls; $n=2$ for individual stimulations. BCR: B-cell receptor stimulation; CD4OL: CD40 pathway stimulation by the CD40 ligand; CpG: stimulation of Toll-like receptor signaling by the dinucleotide CpG.

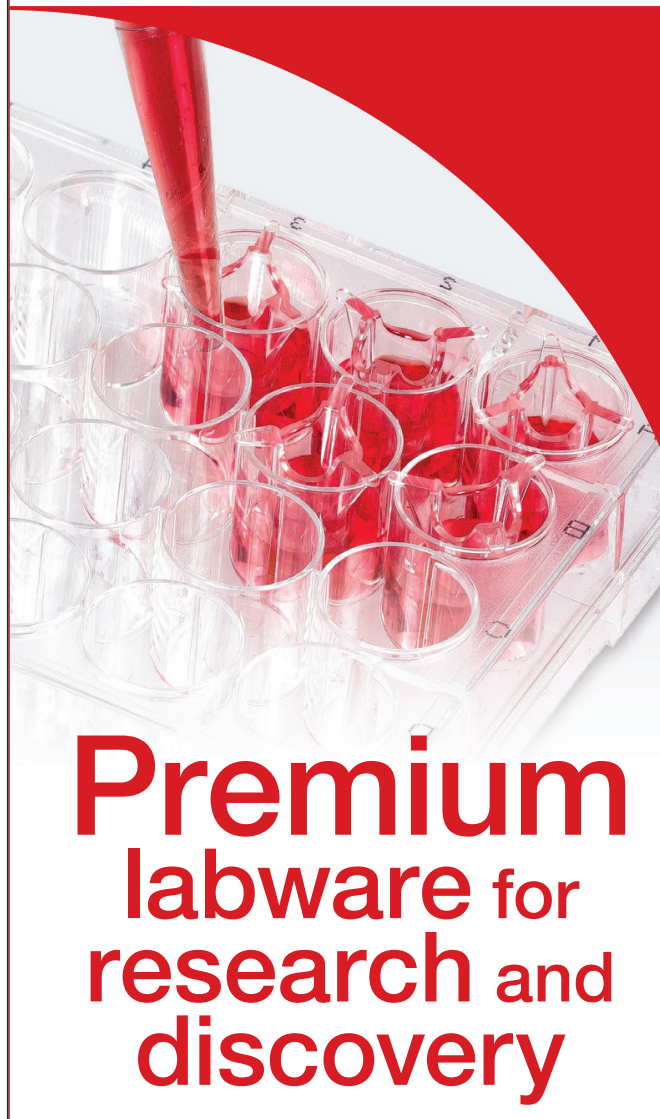

TC Inserts

Hanging inserts for TC plates

In combination with our plates, the inserts form a 2-compartment cell culture system for a wide range of experiments!

- Options for 6, 12 and 24 well plates

- Five different pore sizes from 0.4 to $8 \mu \mathrm{m}$

- Ultra-thin transparent or translucent membrane
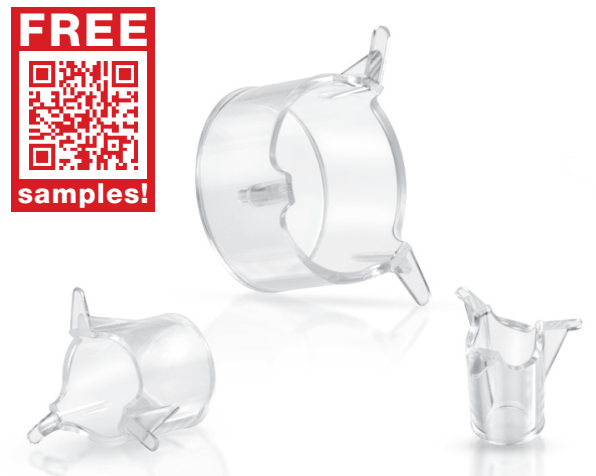

www.sarstedt.com 
type fr-unstranded) and default settings for the remaining parameters.

Normalization and differential gene expression analysis

Read counts were assigned to Ensembl gene identifiers using featureCounts version 1.4 .5 (15). For all data sets, we selected human genes with $>100$ counts in each sample for normalization and differential gene expression analyses. Of the Drosophila genes and ERCC transcripts, the ones with a sum of at least 100 counts over all samples were kept. In the dilution data set, all genes with zero counts in at least one sample were removed. This resulted in 10028 human Ensembl genes, 6070 Drosophila Ensembl genes, and 43 ERCC transcripts being included in the dilution data set. In the MYC data set, 8477 human Ensembl genes, 7050 Drosophila Ensembl genes, and 52 ERCC transcripts were analyzed.

Normalization based on endogenous (human) genes was performed using size factors calculated by DESeq2 (16), followed by log2 fold-change estimates between sample groups. To estimate log2 fold changes based on the Drosophila spike-in cells, size factors were calculated on the counts of the Drosophila genes and applied to the human gene counts prior to log2 fold change estimation with DESeq2 (see protocol available online). For calibration with the ERCC synthetic gene counts, size factors were calculated as described above and applied to the human gene counts followed by log2 fold-change estimation with DESeq2. Fold changes of human genes meeting the count cutoff above were included in the figures, irrespective of their $P$-values from the Wald test as implemented in DESeq2. For Table 1, $P$-values from the Wald test were adjusted to control the false discovery rate (FDR) based on the algorithm by Benjamini and Hochberg (17) implemented in the p.adjust function of the R package 'stats' (www.R-project.org).

\section{Results and discussion}

\section{Discriminating human and}

Drosophila transcripts

The transcripts of the spike-in organism and the organism under study should be sufficiently different to minimize assigning reads to the wrong organism. The D. melanogaster genome has a high-quality sequence assembly, and the sequence divergence should be large enough to discriminate between human and Drosophila transcripts, while the lysis properties of the Drosophila and human cells used in this study should be similar. With our assay, cells from both organisms are lysed and sequenced together, and the resulting libraries are mapped to the concatenated genomes of both organisms. To validate the hypothesis that human and Drosophila reads can be reliably separated, we obtained two libraries each for $D$. melanogaster $S 2$ cells (SRR569914, SRR424185) and human P493-6 cells (SRR567561, SRR567562) from the Sequence Read Archive (SRA). We first mapped the human libraries to the human genome alone and then to the concatenated genome of both organisms. The number of human multi-mapped reads increased from $85,298,194$ to $85,324,091$ and from $84,178,617$ to $84,205,272$ for the 2 RNA-Seq libraries, when using only the human versus the concatenated genome. This corresponds to an increase of $<0.02 \%$ for both libraries. Thus, the fraction of human reads that can be uniquely mapped to the human genome can be reliably identified as human, and these reads are not lost by adding Drosophila sequences. Next, we mapped the Drosophila libraries to the concatenated genome to see whether Drosophila sequences could falsely be assigned to the human genome. Less than 0.01\% of the Drosophila reads mapped
A

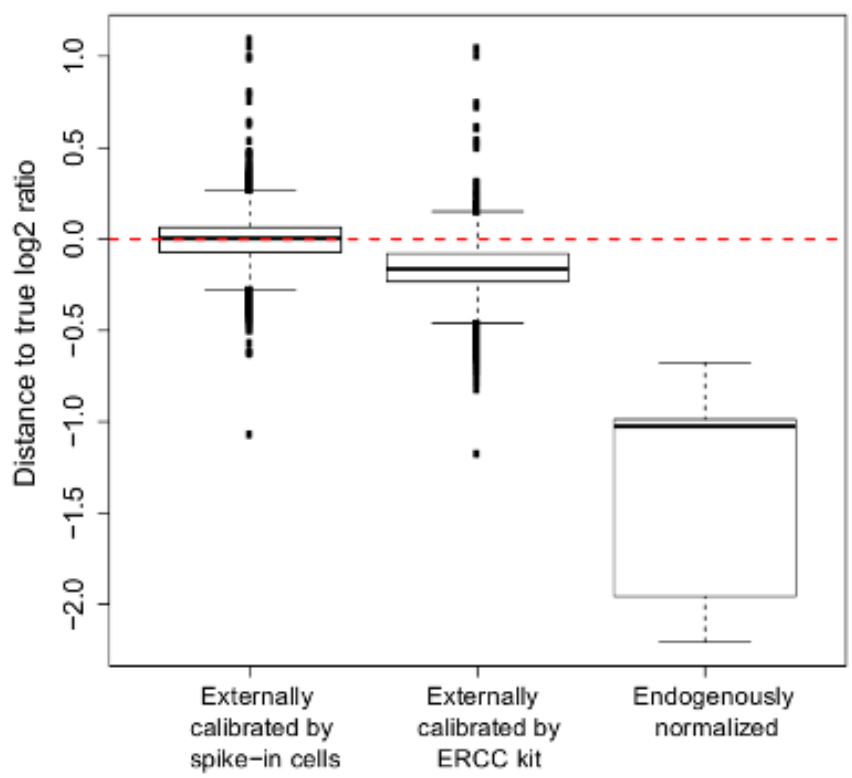

B

RNA-seq
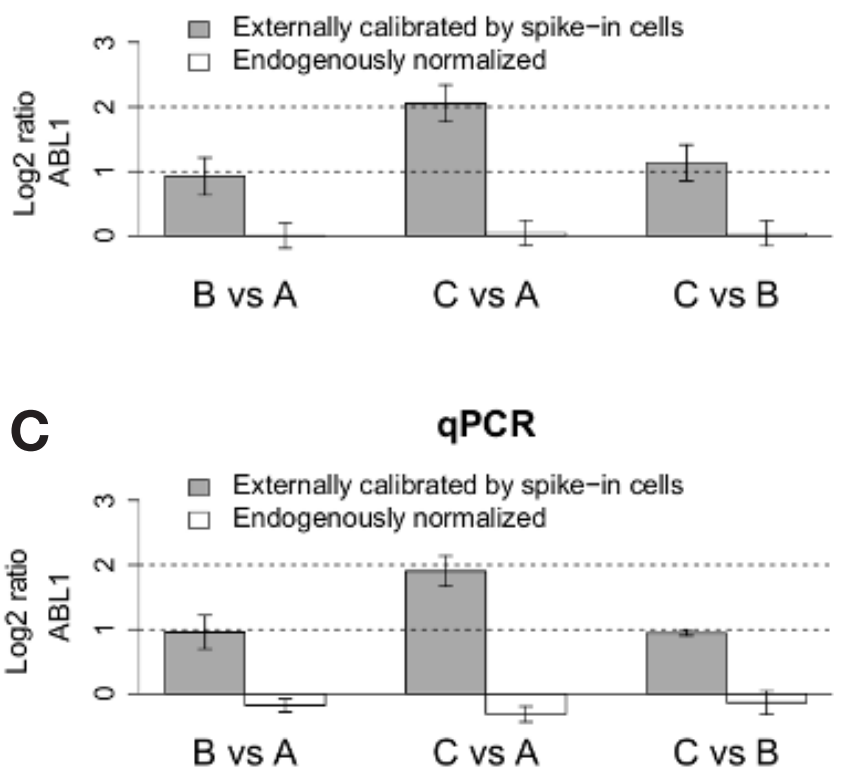

Figure 2. External calibration using Drosophila spike-in cells recovers the true gene expression ratios in a dilution experiment. (A) Bias from true log2 ratios after calibrating the human gene expression data to the Drosophila spike-in data (left), after calibrating to ERCC synthetic RNA spike-in data (center), and after endogenous normalization (relative to a fixed-weight human RNA aliquot, right). The dashed line shows perfect agreement of the expected and estimated log2 ratios (distance equal to zero). (B) Ratios of $A B L 1$ gene expression based on RNA-Seq data. Gray: calibrated to Drosophila spikein cells; white: endogenous normalization (relative to a fixed-weight RNA aliquot). (C) Ratios of $A B L 1$ gene expression based on qPCR data calibrated to Drosophila Act42A (spike-in) or human GAPDH housekeeping gene expression levels. Dashed lines show true log2 ratios between sample groups. 
uniquely to the human part, showing that Drosophila reads are not confused for human sequences with high frequency. Taken together, cross-mapping of reads to the wrong organism can be assumed to be negligible, establishing Drosophila as a workable spike-in organism for human expression profiling with RNA-Seq.

Calibrating gene expression values to the number of sample cells

Next, we demonstrated that Drosophila spike-in cells can be used to change the reference point to a fixed number of sample cells, thereby revealing non-symmetric gene expression changes in RNA-Seq and qPCR data. We simulated variable amounts of total RNA in a fixed number of cells with a dilution experiment. Three groups of human P493-6 cells (A: 500,000 cells, B: 1 million cells, and $\mathrm{C}$ : 2 million cells) were each mixed with 100,000 freshly thawed Drosophila S2 cells (Figure 1A). The mixed cell pools were lysed, RNA was extracted in equal amounts of buffer, and ERCC spike-in RNAs were added. This experimental setup simulated increasing amounts of RNA with defined ratios between the sample groups. We expected ratios of 1:2 in the $A$ versus $B$ and $B$ versus $C$ comparisons, and a ratio of $1: 4$ in the $A$ versus C comparison (Figure 1B). The fixedweight RNA aliquot subjected to library preparation and sequencing consisted of transcripts of human and Drosophila origin, and after mapping the reads to the concatenated genome of both organisms, the percentage of reads mapping to Drosophila genes inversely correlated with the amount of human total RNA in the sample (Figure 1C). The human gene expression profiles were then calibrated to constant Drosophila gene expression levels. This restored the original ratios between the conditions (Figure 1D).

As an alternative method to externally calibrate the data, we derived size factors from the ERCC synthetic RNA spike-ins. In addition, we endogenously normalized the human expression data to a fixed-weight aliquot of RNA. Since cells were taken from the same population, the RNA composition should have been constant across all of the samples. Thus, we expected a ratio of 1:1 between all genes or, equivalently, a difference of 0 for log2 ratios of the endogenously normalized data.
Figure 2A shows differences of estimated log2 ratios for Drosophila externally calibrated data (left), externally calibrated data using ERCC spike-ins (center), and endogenously normalized count data (right) from the ratios expected based on the experimental design. Externally calibrated data (Figure 2A, left and middle) reflect the expected ratios, and correspond to ratios of absolute abundances. Comparing external calibration by Drosophila cells (Figure 2A, left) and ERCC RNA (Figure 2A, middle), calibration with Drosophila cells led to deviations from the true ratio scattering around zero, while calibration with the ERCC RNA led to a small but systematic bias in recovering the true ratio. Drosophila calibrated gene expression data has lower noise in log2 fold-change estimates compared with data calibrated with the ERCC spike-ins $(P<2.2 \times$ $10^{-16}$, Levene's test for homogeneity of variance). The log2 ratios of the endogenously normalized data (Figure 2A, right) scatter around zero, hence the differences to the true log2 ratios scatter around one and two. This observation indicates that the addition of the spike-in cells did not distort the estimation of relative expression values for human cells. That is, relative fold changes can be estimated from the same data when the spike-in data are ignored, and the data can be analyzed with multiple reference points.
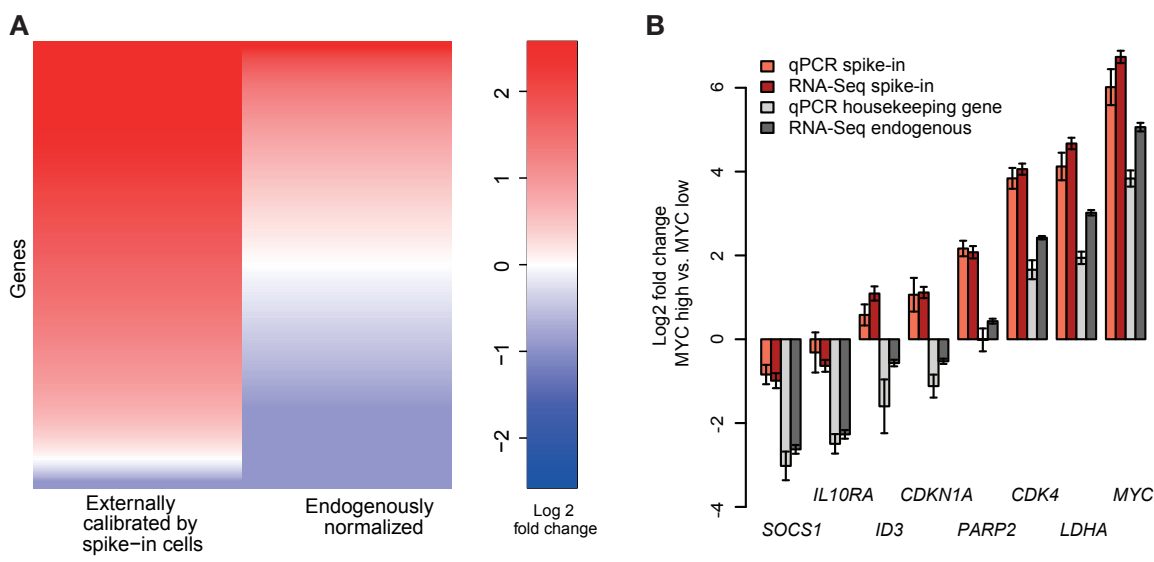

Figure 3. Gene expression in the human P493-6 cell line with different levels of MYC. (A) Log2 gene expression fold changes between cells expressing high levels of MYC and cells expressing low levels of MYC calculated after calibrating the human RNA-Seq data with the Drosophila spike-in cell data (externally calibrated, left) and without calibration to the external standards (endogenously normalized, that is, relative to a fixed-weight RNA aliquot, right) $(n=10)$. (B) Gene expression levels quantified with GPCR calibrated to a Drosophila spike-in gene (light red), quantified with RNA-Seq and calibrated to Drosophila gene expression (dark red), quantified with GPCR and calibrated to an endogenous housekeeping gene (human GAPDH, light gray), or quantified with RNA-Seq and endogenously normalized (dark gray). Means and standard errors (SE) of 3 (qPCR) and 10 (RNA-Seq) independent biological replicates are shown. 
Externally calibrated by spike-in cells

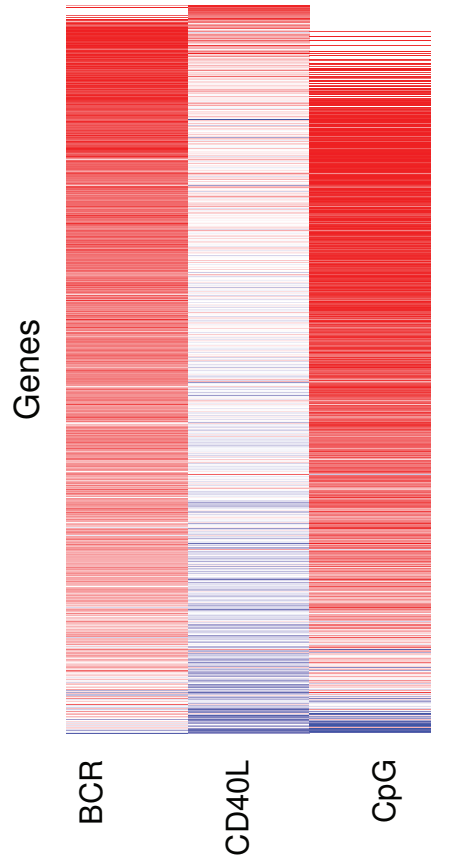

\section{Endogenously} normalized

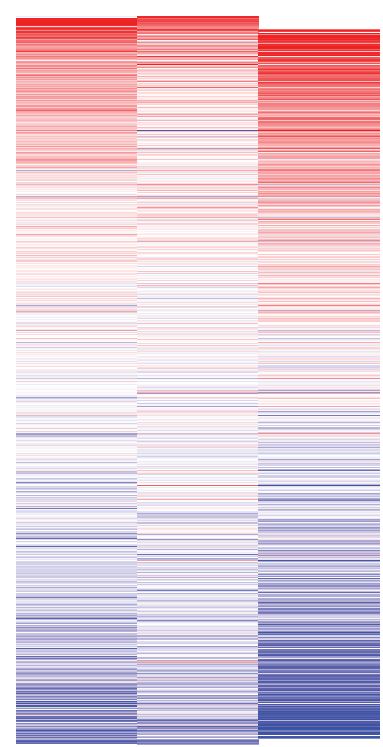

$\stackrel{\Upsilon}{\stackrel{\Upsilon}{0}}$

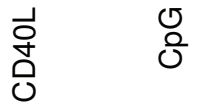

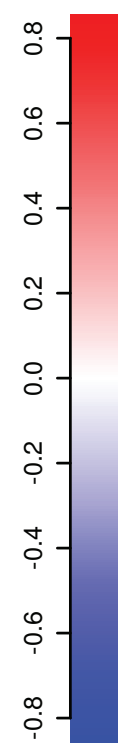

$\log 2$ fold change

Figure 4. Log 2 fold changes of gene expression after treatment of the human P493-6 cell line with various physiological stimuli. Left: Gene expression data externally calibrated to the number of cells in the sample using Drosophila spike-in cells. Right: endogenously normalized gene expression values (relative to a fixed-weight RNA aliquot). BCR: B-cell receptor stimulation; CD40 pathway stimulation by the CD40 ligand (CD4OL); CpG: stimulation of Toll-like receptor signaling by the dinucleotide CpG. $n=10$ for unstimulated controls; $n=2$ for individual stimulations.

In addition, Drosophila spike-in cells are cheap to maintain (Supplementary Table S2). Once purchased, they can easily be amplified by cell cultivation. On the other hand, constant cell cultivation is not needed once the cells are frozen, since they can then be taken directly from a frozen stock and spiked into the sample. Within a set of experiments, cells from the same freezing stock should be used to ensure a homogeneous cell population.

\section{Assessing non-symmetric global gene expression changes in response to MYC induction}

MYC protein up-regulates the transcription of almost all actively transcribed genes in the human B-cell line P493-6 (5). We analyzed MYC-driven global transcriptional up-regulation using our Drosophila spike-in assay with RNA-Seq and qPCR. Comparing gene expression levels before and after induction of the ectopic MYC gene using spike-in calibration, global induction of gene expression was evident (Figure 3A). Endogenously normalizing the same data, log2 fold changes scattered around zero, indicating similar numbers of induced and repressed genes (Figure

3A). The amount of total RNA extracted from the same number of cells differed by a factor of nearly 1.8 between Myc ${ }^{\text {high }}$ and $\mathrm{Myc}^{\mathrm{low}}$ samples (Supplementary Figure S1).

We also analyzed the expression levels of eight human genes (IL1ORA, SOCS1, ID3, CDKN1A, PARP2 CDK4, $\angle D H A, M Y C)$ using GPCR with two different reference genes: (i) the human housekeeping gene GAPDH and (ii) the Drosophila gene Act42A. Figure $3 \mathrm{~B}$ shows the corresponding log2 fold changes in response to MYC induction. Calibration to the Drosophila reference gene indicated that 6 out of 8 genes were induced in Mychigh cells and only 2 were repressed, while calibration to the endogenous reference gene suggested that approximately half of the genes analyzed were induced, while the other half were repressed. Therefore, global changes in gene expression also impact conventional aPCR measurements. The fold changes resulting from the GPCR measurements were consistent with the fold changes from the RNA-Seq data for external calibration based on Drosophila gene expression, while the fold changes based on endogenous normalization from RNA-Seq showed a positive shift compared with the ones from the GPCR data (Figure 3B). The latter is most likely due to the difference in reference points: the RNA-Seq data were normalized to a fixed-weight aliquot of RNA, while the qPCR data were normalized to a single reference gene.

To determine if perturbations less extreme than the ectopic expression of MYC could also lead to non-symmetric gene expression changes, we treated P493-6 Myclow cells with different B-cell stimuli. These included: (i) activation of Toll-like receptor signaling by the dinucleotide CpG; (ii) activation of the CD40 pathway by the CD40 ligand (CD4OL), and (iii) activation of $\mathrm{B}$-cell receptor (BCR) signaling via crosslinking of the $\mathrm{BCR}$ using $\alpha$-IgM F(ab)2 fragments. When the data were normalized to endogenous genes, similar numbers of induced and repressed genes were observed after each type of B-cell stimulation. When the data were calibrated to the Drosophila genes, strong induction of many genes after BCR and CpG stimulation became evident (Figure 4, Table 1). CD4OL induced small and nearly balanced gene expression changes of a limited number of genes (Figure 4), which were not significant when the data were externally calibrated (Table 1). Exogenous calibration to Drosophila cells revealed that global gene expression changes do also occur under physiological conditions in B cells, while endogenous calibration suggested equal numbers of induced and repressed genes after B-cell activation.

Having estimated absolute fold changes based on Drosophila spike-ins, it might still be of interest to determine which genes were induced even more than the average global transcriptional change and which participated the least in the global transcriptional change. These gene lists will be similar to the ones generated based on relative fold-change estimates, but we suggest to interpret them relative to the global change, not the fixed RNA amount. From relative fold changes alone, we cannot reconstruct whether the gene was actively repressed or appears repressed as a compensatory artifact associated with this reference point. Since the induction and repression of gene expression are likely caused by different molecular mechanisms, we strongly recommend 
using spike-in protocols and calibration to total cell numbers for interpreting gene regulation mechanistically.

\section{Author contributions}

F.T. performed sequence data analyses, generated figures, and contributed to manuscript writing. M.F. performed the experiments, analyzed data, and contributed to manuscript writing. P.S. and W.G. contributed to the conception and design of the study and critically revised the manuscript. D.K. coordinated the experimental part of the study. D.K., R.S., and J.C.E. conceived and coordinated this study. R.S. and J.C.E. wrote the manuscript. All authors read and approved the final manuscript before submission.

\section{Acknowledgments}

This work was funded by the e:Bio initiative of the German Federal Ministry of Education and Research (BMBF), grant numbers 0316166G and 0316166E. We thank Georg Bornkamm (Munich) for providing P493-6 cells.

\section{Competing interests}

The authors declare no competing interests.

\section{References}

1. Bolstad, B.M., R.A. Irizarry, M. Astrand, and T.P. Speed. 2003. A comparison of normalization methods for high density oligonucleotide array data based on variance and bias. Bioinformatics 19:185-193.

2. Reimers, M. 2010. Making informed choices about microarray data analysis. PLOS Comput. Biol. 6:e1000786.

3. Buettner, F., K.N. Natarajan, F.P. Casale, V. Proserpio, A. Scialdone, F.J. Theis, S.A. Teichmann, J.C. Marioni, and O. Stegle. 2015. Computational analysis of cell-to-cell heterogeneity in single-cell RNA-Sequencing data reveals hidden subpopulations of cells. Nat. Biotechnol. 33:155-160.

4. Lin, C.Y., J. Lovén, P.B. Rahl, R.M. Paranal, C.B. Burge, J.E. Bradner, T.I. Lee, and R.A. Young. 2012. Transcriptional Amplification in Tumor Cells with Elevated c-Myc. Cell 151:56-67.

5. Nie, Z., G. Hu, G. Wei, K. Cui, A. Yamane, W. Resch, R. Wang, D.R. Green, et al. 2012. C-Myc Is a Universal Amplifier of Expressed Genes in Lymphocytes and Embryonic Stem Cells. Cell 151:68-79.

6. Sabò, A., T.R. Kress, M. Pelizzola, S. de Pretis, M.M. Gorski, A. Tesi, M.J. Morelli, P. Bora, et al. 2014. Selective transcriptional regulation by Myc in cellular growth control and lymphomagenesis. Nature 511:488-492.

7. van de Peppel, J., P. Kemmeren, H. van Bakel, M. Radonjic, D. van Leenen, and F.C.P.
Holstege. 2003. Monitoring global messenger RNA changes in externally controlled microarray experiments. EMBO Rep. 4:387-393.

8. Chen, K., Z. Hu, Z. Xia, D. Zhao, W. Li, and J.K. Tyler. 2015. The overlooked fact: fundamental need for spike-in control for virtually all genome-wide analyses. Mol. Cell. Biol. 36:662667.

9. Lovén, J., D.A. Orlando, A.A. Sigova, C.Y Lin, P.B. Rahl, C.B. Burge, D.L. Levens, T.I. Lee, and R.A. Young. 2012. Revisiting Global Gene Expression Analysis. Cell 151:476-482.

10. Brennecke, P., S. Anders, J.K. Kim, A.A. Kołodziejczyk, X. Zhang, V. Proserpio, B. Baying, V. Benes, et al. 2013. Accounting for technical noise in single-cell RNA-Seq experiments. Nat. Methods 10:1093-1095.

11. Sun, M., B. Schwalb, D. Schulz, N. Pirkl, S. Etzold, L. Larivière, K.C. Maier, M. Seizl, et al. 2012. Comparative dynamic transcriptome analysis (cDTA) reveals mutual feedback between mRNA synthesis and degradation. Genome Res. 22:1350-1359.

12. Baker, S.C., S.R. Bauer, R.P. Beyer, J.D. Brenton, B. Bromley, J. Burrill, H. Causton, M.P. Conley, et al. 2005. The External RNA Controls Consortium: a progress report. Nat. Methods 2:731-734.

13. Cunningham, F., M.R. Amode, D. Barrell, K. Beal, K. Billis, S. Brent, D. Carvalho-Silva, P.
Clapham, et al. 2015. Ensembl 2015. Nucleic Acids Res. 43(D1):D662-D669.

14. Kim, D., G. Pertea, C. Trapnell, H. Pimentel, R. Kelley, and S.L. Salzberg. 2013. TopHat2: accurate alignment of transcriptomes in the presence of insertions, deletions and gene fusions. Genome Biol. 14:R36.

15. Liao, Y., G.K. Smyth, and W. Shi. 2014. featureCounts: an efficient general purpose program for assigning sequence reads to genomic features. Bioinformatics 30:923-930.

16. Love, M.I., W. Huber, and S. Anders. 2014. Moderated estimation of fold change and dispersion for RNA-Seq data with DESeq2. Genome Biol. 15:550.

17. Benjamini, Y. and Y. Hochberg. 1995. Controlling the false discovery rate: a practical and powerful approach to multiple testing. J. R. Stat. Soc. Series B Stat. Methodol. 57:289-300.

Received 10 August 2016; accepted 17 November 2016.

Address correspondence to Julia C. Engelmann, Statistical Bioinformatics, University of Regensburg, Am BioPark 9, 93053 Regensburg, Germany. Email: julia.engelmann@ur.de

To purchase reprints of this article, contact: biotechniques@fosterprinting.com

\section{BEAD RUPTOR ELITE Bead Mill Homogenizer}

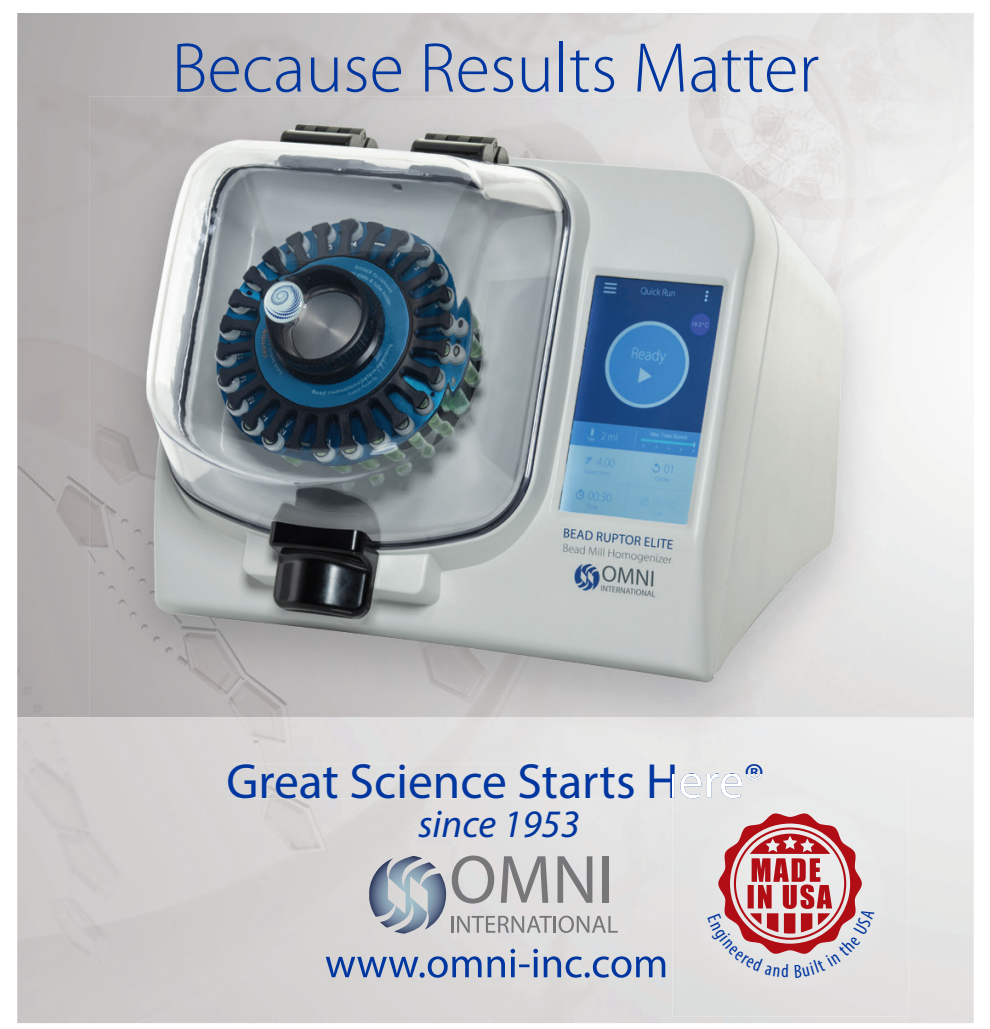

